\title{
A Distributed Quasi-Newton Algorithm for Empirical Risk Minimization with Nonsmooth Regularization
}

\author{
Ching-pei Lee \\ Department of Computer Sciences \\ University of Wisconsin-Madison \\ Madison, Wisconsin \\ ching-pei@cs.wisc.edu
}

\author{
Cong Han Lim \\ Wisconsin Institute for Discovery \\ University of Wisconsin-Madison \\ Madison, Wisconsin \\ clim9@wisc.edu
}

\author{
Stephen J. Wright \\ Department of Computer Sciences \\ University of Wisconsin-Madison \\ Madison, Wisconsin \\ swright@cs.wisc.edu
}

\begin{abstract}
We propose a communication- and computation-efficient distributed optimization algorithm using second-order information for solving ERM problems with a nonsmooth regularization term. Current second-order and quasi-Newton methods for this problem either do not work well in the distributed setting or work only for specific regularizers. Our algorithm uses successive quadratic approximations, and we describe how to maintain an approximation of the Hessian and solve subproblems efficiently in a distributed manner. The proposed method enjoys global linear convergence for a broad range of non-strongly convex problems that includes the most commonly used ERMs, thus requiring lower communication complexity. It also converges on non-convex problems, so has the potential to be used on applications such as deep learning. Initial computational results on convex problems demonstrate that our method significantly improves on communication cost and running time over the current state-of-the-art methods.
\end{abstract}

\section{CCS CONCEPTS}

- Computing methodologies $\rightarrow$ Machine learning algorithms; Distributed algorithms;

\section{KEYWORDS}

Distributed optimization, Empirical risk minimization, Nonsmooth optimization, Regularized optimization, Variable metrics, Quasi-Newton, Proximal method, Inexact method

\section{ACM Reference Format:}

Ching-pei Lee, Cong Han Lim, and Stephen J. Wright. 2018. A Distributed Quasi-Newton Algorithm for Empirical Risk Minimization with Nonsmooth Regularization. In KDD '18: The 24th ACM SIGKDD International Conference on Knowledge Discovery E Data Mining, August 19-23, 2018, London, United Kingdom.

Permission to make digital or hard copies of all or part of this work for personal or classroom use is granted without fee provided that copies are not made or distributed for profit or commercial advantage and that copies bear this notice and the full citation on the first page. Copyrights for components of this work owned by others than the author(s) must be honored. Abstracting with credit is permitted. To copy otherwise, or republish, to post on servers or to redistribute to lists, requires prior specific permission and/or a fee. Request permissions from permissions@acm.org.

KDD '18, August 19-23,2018, London, United Kingdom

(C) 2018 Copyright held by the owner/author(s). Publication rights licensed to ACM

ACM ISBN 978-1-4503-5552-0/18/08 . .\$15.00

https://doi.org/10.1145/3219819.3220075
ACM, New York, NY, USA, 10 pages. https://doi.org/10.1145/ 3219819.3220075

\section{INTRODUCTION}

We consider solving the following regularized problem in a distributed manner:

$$
\min _{\boldsymbol{w} \in \mathbb{R}^{d}} F(\boldsymbol{w}):=f\left(X^{T} \boldsymbol{w}\right)+g(\boldsymbol{w}),
$$

where $X$ is a $d$ by $n$ real-valued matrix, $g$ is a convex, closed, and extended-valued proper function that can be nondifferentiable, and $f$ is a differentiable function whose gradient is Lipschitz continuous with parameter $L>0$. Each column of $X$ represents a single data point or instance, and we assume that the set of data points is partitioned and spread across $K$ machines (i.e. distributed instance-wise). We can write $X$ as

$$
X:=\left[X_{1}, X_{2}, \ldots, X_{K}\right],
$$

where $X_{k}$ is stored exclusively on the $k$ th machine. We further assume that $f$ shares the same block-separable structure and can be written as follows:

$$
f\left(X^{T} \boldsymbol{w}\right)=\sum_{k=1}^{K} f_{k}\left(X_{k}^{T} \boldsymbol{w}\right) .
$$

Unlike our instance-wise setting, some existing works consider the feature-wise partition setting, under which $X$ is partitioned by rows rather than columns. Although the featurewise setting is a simpler one for algorithm design when $g$ is separable, storage of different features on different machines is often impractical.

The bottleneck in performing distributed optimization is often the high cost of communication between machines. For (1), the time required to retrieve $X_{k}$ over a network can greatly exceed the time needed to compute $f_{k}$ or its gradient with locally stored $X_{k}$. Moreover, we incur a delay at the beginning of each round of communication due to the overhead of establishing connections between machines. This latency prevents many efficient single-core algorithms such as coordinate descent (CD) and stochastic gradient and their asynchronous parallel variants from being employed in large-scale distributed computing setups. Thus, a key aim of algorithm design for distributed optimization is to improve the communication efficiency while keeping the computational cost affordable. Batch methods are preferred in this context, because fewer rounds of communication occur in distributed batch methods. 
When $F$ is differentiable, many efficient batch methods can be used directly in distributed environments to solve (1). For example, Nesterov's accelerated gradient (AG) [16] enjoys low iteration complexity, and since each iteration of AG only requires one round of communication to compute the new gradient, it also has good communication complexity. Although its supporting theory is not particularly strong, the limited-memory BFGS (LBFGS) method [13] is popular among practitioners of distributed optimization. It is the default algorithm for solving $\ell_{2}$-regularized smooth ERM problems in Apache Spark's distributed machine learning library [14], as it is empirically much faster than AG (see, for example, the experiments in Wang et al. [22]). Other modified batch methods that utilize the Hessian of the objective in various ways are also communication-efficient under their own additional assumptions [7, 12, 20, 26, 28].

When $g$ is nondifferentiable, neither LBFGS nor Newton's method can be applied directly. Leveraging curvature information from $f$ can still be beneficial in this setting. For example, the orthant-wise quasi-Newton method OWLQN [2] adapts the LBFGS algorithm to the special nonsmooth case in which $g(\cdot) \equiv\|\cdot\|_{1}$, and is popular for distributed optimization of $\ell_{1}$-regularized ERM problems. Extension of this approach to other nonsmooth $g$ is not well understood, and the convergence guarantees are only asymptotic, rather than global.

To the best of our knowledge, for ERMs with general nonsmooth regularizers in the instance-wise storage setting, proximal-gradient-like methods $[17,23]$ are the only practical distributed optimization algorithms with convergence guarantees. Since these methods barely use the Hessian information of the smooth part (if at all), we suspect that proper utilization of second-order information has the potential to improve convergence speed and therefore communication efficiency dramatically. We thus propose a practical distributed inexact variable-metric algorithm for general (1) which uses gradients and which updates information from previous iterations to estimate curvature of the smooth part $f$ in a communication-efficient manner. We describe construction of this estimate and solution of the corresponding subproblem. We also provide convergence rate guarantees, which also bound communication complexity. These rates improve on existing distributed methods, even those tailor-made for specific regularizers.

Our algorithm leverages the more general framework provided in Lee and Wright [9], and our major contribution in this work is to describe how the main steps of the framework can be implemented efficiently in a distributed environment. Our approach has both good communication and computational complexity, unlike certain approaches that focus only on communication at the expense of computation (and ultimately overall time). We believe that this work is the first to propose, analyze, and implement a practically feasible distributed optimization method for solving (1) with general nonsmooth regularizer $g$ under the instance-wise storage setting.
Our algorithm and implementation details are given in Section 2. Communication complexity and the effect of the subproblem solution inexactness are analyzed in Section 3. Section 4 discusses related works, and empirical comparisons are conducted in Section 5. Concluding observations appear in Section 6.

\section{Notation}

We use the following notation.

- $f\left(X^{T} \boldsymbol{w}\right)$ is abbreviated as $\tilde{f}(\boldsymbol{w})$.

- $\|\cdot\|$ denotes the 2-norm, both for vectors and for matrices.

- Given any symmetric positive semi-definite matrix $H \in$ $\mathbb{R}^{d \times d}$ and any vector $\boldsymbol{p} \in \mathbb{R}^{d},\|\boldsymbol{p}\|_{H}$ denotes the semi-norm $\sqrt{\boldsymbol{p}^{T} H \boldsymbol{p}}$.

\section{ALGORITHM}

At each iteration of our algorithm for optimizing (1), we construct a subproblem that consists of a quadratic approximation of $\tilde{f}$ added to the original regularizer $g$. Specifically, given the current iterate $\boldsymbol{w}$, we choose a positive semi-definite $H$ and define

$$
Q_{H}(\boldsymbol{p} ; \boldsymbol{w}):=\nabla \tilde{f}(\boldsymbol{w})^{T} \boldsymbol{p}+\frac{1}{2}\|\boldsymbol{p}\|_{H}^{2}+g(\boldsymbol{w}+\boldsymbol{p})-g(\boldsymbol{w}),
$$

the update direction is obtained by approximately solving

$$
\min _{\boldsymbol{p} \in \mathbb{R}^{d}} Q_{H}(\boldsymbol{p} ; \boldsymbol{w}) .
$$

A line search procedure determines a suitable stepsize $\alpha$, and we perform the update $\boldsymbol{w} \leftarrow \boldsymbol{w}+\alpha \boldsymbol{p}$.

We now discuss the following issues in the distributed setting: communication cost, the computation of $\nabla \tilde{f}$, the choice and construction of $H$, procedures for solving (2), and the line search procedure. In our description, we sometimes need to split some $n$-dimensional vectors over the machines, in accordance with the following disjoint partition $J_{1}, \ldots, J_{k}$ of $\{1, \ldots, d\}$ :

$$
J_{i} \cap J_{k}=\phi, \forall i \neq k, \quad \cup_{i=1}^{K} J_{i}=\{1, \ldots, d\} .
$$

\subsection{Communication Cost}

For the ease of description, we assume the allreduce model of MPI [15], but it is also straightforward to extend the framework to a master-worker platform. Under this model, all machines simultaneously fulfill master and worker roles, and any data transmitted is broadcast to all machines. This can be considered as equivalent to conducting one map-reduce operation and then broadcasting the result to all nodes. The communication cost for the allreduce operation on a $d$-dimensional vector under this model is

$$
\log (K) T_{\text {initial }}+d T_{\text {byte }},
$$

where $T_{\text {initial }}$ is the latency to establish connection between machines, and $T_{\text {byte }}$ is the per byte transmission time (see, for example, Chan et al. [5, Section 6.3]).

The first term in (3) also explains why batch methods are preferable. Even if methods that frequently update the iterates communicate the same amount of bytes, it takes more rounds of communication to transmit the information, 
and the overhead of $\log (K) T_{\text {initial }}$ incurred at every round of communication makes this cost dominant, especially when $K$ is large.

In subsequent discussion, when an allreduce operation is performed on a vector of dimension $O(d)$, we simply say that $O(d)$ communication is conducted. We omit the latency term since batch methods like ours tend to have only a small constant number of rounds of communication per iteration. By contrast, non-batch methods such as CD or stochastic gradient require $O(d)$ or $O(n)$ rounds of communication per epoch and therefore face much more significant latency issues.

\subsection{Computing $\nabla \tilde{f}$}

The gradient of $\tilde{f}$ has the form

$$
\nabla \tilde{f}(\boldsymbol{w})=X \nabla f\left(X^{T} \boldsymbol{w}\right)=\sum_{k=1}^{K}\left(X_{k} \nabla f_{k}\left(X_{k}^{T} \boldsymbol{w}\right)\right) .
$$

We see that, except for the sum over $k$, the computation can be conducted locally provided $\boldsymbol{w}$ is available to all machines. Our algorithm maintains $X_{k}^{T} \boldsymbol{w}$ on the $k$ th machine throughout, and the most costly steps are the matrix-vector multiplications between $X_{k}$ and $\nabla f_{k}\left(X_{k}^{T} \boldsymbol{w}\right)$, and $X^{T} \boldsymbol{w}$. The local $d$-dimensional partial gradients are then aggregated through an allreduce operation.

\subsection{Constructing a good $H$ efficiently}

We use the Hessian approximation constructed by the LBFGS algorithm [13], and propose a way to maintain and utilize it efficiently in a distributed setting. Using the compact representation in Byrd et al. [4], given a prespecified integer $m>0$, at the $t$ th iteration for $t>0$, let $\tilde{m}:=\min (m, t)$, and define

$$
\boldsymbol{s}_{i}:=\boldsymbol{w}_{i+1}-\boldsymbol{w}_{i}, \quad \boldsymbol{y}_{i}:=\nabla \tilde{f}\left(\boldsymbol{w}_{i+1}\right)-\nabla \tilde{f}\left(\boldsymbol{w}_{i}\right), \quad \forall i .
$$

The LBFGS Hessian approximation matrix is

$$
H_{t}=\gamma_{t} I-U_{t} M_{t}^{-1} U_{t}^{T},
$$

where

$$
\begin{aligned}
U_{t} & :=\left[\gamma_{t} S_{t}, Y_{t}\right], \quad M_{t}:=\left[\begin{array}{cc}
\gamma_{t} S_{t}^{T} S_{t}, & L_{t} \\
L_{t}^{T} & -D_{t}
\end{array}\right], \\
\gamma_{t} & :=\frac{\boldsymbol{s}_{t-1}^{T} \boldsymbol{s}_{t-1}}{\boldsymbol{s}_{t-1}^{T} y_{t-1}}
\end{aligned}
$$

and

$$
\begin{aligned}
S_{t} & :=\left[\boldsymbol{s}_{t-\tilde{m}}, \boldsymbol{s}_{t-\tilde{m}+1}, \ldots, \boldsymbol{s}_{t-1}\right], \\
Y_{t} & :=\left[\boldsymbol{y}_{t-\tilde{m}}, \boldsymbol{y}_{t-\tilde{m}+1}, \ldots, \boldsymbol{y}_{t-1}\right], \\
D_{t} & :=\operatorname{diag}\left(\boldsymbol{s}_{t-\tilde{m}}^{T} \boldsymbol{y}_{t-\tilde{m}}, \ldots, \boldsymbol{s}_{t-1}^{T} \boldsymbol{y}_{t-1}\right), \\
\left(L_{t}\right)_{i, j} & := \begin{cases}\boldsymbol{s}_{t-m-1+i}^{T} \boldsymbol{y}_{t-m-1+j}, & \text { if } i>j, \\
0, & \text { otherwise. }\end{cases}
\end{aligned}
$$

At the first iteration where no $\boldsymbol{s}_{i}$ and $\boldsymbol{y}_{i}$ are available, we set $H_{0}:=a_{0} I$ for some positive scalar $a_{0}$. When $f$ is twicedifferentiable and convex, we use

$$
a_{0}:=\frac{\left\|\nabla f\left(\boldsymbol{w}_{0}\right)\right\|_{\nabla^{2} f\left(\boldsymbol{w}_{0}\right)}^{2} .}{\left\|\nabla f\left(\boldsymbol{w}_{0}\right)\right\|^{2}} .
$$

If $f$ is not strongly convex, it is possible that (5) is only positive semi-definite. In this case, we follow $\mathrm{Li}$ and Fukushima [11], taking the $m$ update pairs to be the most recent $m$ iterations for which the inequality

$$
\boldsymbol{s}_{i}^{T} \boldsymbol{y}_{i} \geq \delta \boldsymbol{s}_{i}^{T} \boldsymbol{s}_{i}
$$

is satisfied, for some predefined $\delta>0$. It can be shown that this safeguard ensures that $H_{t}$ are always positive definite and the eigenvalues are bounded within a positive range (see, for example, the appendix of Lee and Wright [8]).

No additional communication is required to compute $H_{t}$. The gradients at all previous iterations have been shared with all machines through the allreduce operation, and the iterates $\boldsymbol{w}_{t}$ are also available on each machine, as they are needed to compute the local gradient. Thus the information needed to form $H_{t}$ is available locally on each machine.

We now consider the costs associated with the matrix $M_{t}$. In practice, $m$ is usually much smaller than $d$, so the $O\left(\mathrm{~m}^{3}\right)$ cost of inverting the matrix directly is insignificant compared to the cost of the other steps. However, if $d$ is large, the computation of the inner products $\boldsymbol{s}_{i}^{T} \boldsymbol{y}_{j}$ and $\boldsymbol{s}_{i}^{T} \boldsymbol{s}_{j}$ can be expensive. We can significantly reduce this cost by computing and maintaining the inner products in parallel and assembling the results with $O(m)$ communication cost. At the $t$ th iteration, given the new $\boldsymbol{s}_{t-1}$, we compute its inner products with both $S_{t}$ and $Y_{t}$ in parallel via the summations

$$
\sum_{k=1}^{K}\left(\left(S_{t}\right)_{J_{k},:}^{T}\left(\boldsymbol{s}_{t-1}\right)_{J_{k}}\right), \quad \sum_{k=1}^{K}\left(\left(Y_{t}\right)_{J_{k},:}^{T}\left(\boldsymbol{s}_{t-1}\right)_{J_{k}}\right),
$$

requiring $O(m)$ communication of the partial sums on each machine. We keep these results until $\boldsymbol{s}_{t-1}$ and $\boldsymbol{y}_{t-1}$ are discarded, so that at each iteration, only $2 m$ (not $O\left(m^{2}\right)$ ) inner products are computed.

\subsection{Solving the Subproblem}

The approximate Hessian $H_{t}$ is generally not diagonal, so there is no easy closed-form solution to (2). We will instead use iterative algorithms to obtain an approximate solution to this subproblem. In single-core environments, coordinate descent (CD) is one of the most efficient approaches for solving (2) [18, 25, 27]. Since the subproblem (2) is formed locally on all machines, a local CD process can be applied when $g$ is separable and $d$ is not too large. The alternative approach of applying proximal-gradient methods to (2) may be more efficient in distributed settings, since they can be parallelized with little communication cost for large $d$, and can be applied to larger classes of regularizers $g$.

The fastest proximal-gradient-type methods are accelerated gradient (AG) [17] and SpaRSA [23]. SpaRSA is a basic proximal-gradient method with spectral initialization of the 
parameter in the prox term. SpaRSA has a few key advantages over AG despite its weaker theoretical convergence rate guarantees. It tends to be faster in the early iterations of the algorithm [24], thus possibly yielding a solution of acceptable accuracy in fewer iterations than AG. It is also a descent method, reducing the objective $Q_{H}$ at every iteration, which ensures that the solution returned is at least as good as the original guess $\boldsymbol{p}=0$

In the rest of this subsection, we will describe a distributed implementation of SpaRSA for (2), with $H$ as defined in (5). To distinguish between the iterations of our main algorithm (i.e. the entire process required to update $\boldsymbol{w}$ a single time) and the iterations of SpaRSA, we will refer to them by main iterations and SpaRSA iterations respectively.

Since $H$ and $\boldsymbol{w}$ are fixed in this subsection, we will write $Q_{H}(\cdot ; \boldsymbol{w})$ simply as $Q(\cdot)$. We denote the $i$ th iterate of the SpaRSA algorithm as $\boldsymbol{p}^{(i)}$, and we initialize $\boldsymbol{p}^{(0)} \equiv 0$. We denote the smooth part of $Q_{H}$ by $\hat{f}(\boldsymbol{p})$, and the nonsmooth $g(\boldsymbol{w}+\boldsymbol{p})$ by $\hat{g}(\boldsymbol{p})$. At the $i$ th iteration of SpaRSA, we define

$$
\mathbf{u}_{\psi_{i}}^{(i)}:=\boldsymbol{p}^{(i)}-\frac{\nabla \hat{f}\left(\boldsymbol{p}^{(i)}\right)}{\psi_{i}},
$$

and solve the following subproblem:

$$
\boldsymbol{p}^{(i+1)}=\arg \min _{\boldsymbol{p}} \frac{1}{2}\left\|\boldsymbol{p}-\mathbf{u}_{\psi_{i}}^{(i)}\right\|^{2}+\frac{\hat{g}(\boldsymbol{p})}{\psi_{i}},
$$

where $\psi_{i}$ is defined by the following "spectral" formula:

$$
\psi_{i}=\frac{\left(\boldsymbol{p}^{(i)}-\boldsymbol{p}^{(i-1)}\right)^{T}\left(\nabla \hat{f}\left(\boldsymbol{p}^{(i)}\right)-\nabla \hat{f}\left(\boldsymbol{p}^{(i-1)}\right)\right)}{\left\|\boldsymbol{p}^{(i)}-\boldsymbol{p}^{(i-1)}\right\|^{2}} .
$$

When $i=0$, we use a pre-assigned value for $\psi_{0}$ instead. (In our LBFGS choice for $H_{t}$, we use the value of $\gamma_{t}$ from (6b) as the initial estimate of $\psi_{0}$.) The exact minimizer of (11) can be difficult to compute for general regularizers $g$. However, approximate solutions of (11) suffice to provide a convergence rate guarantee for solving (2) [6, 9, 18, 19]. Since it is known (see [11]) that the eigenvalues of $H$ are upperand lower-bounded in a positive range after the safeguard (9) is applied, we can guarantee that this initialization of $\psi_{i}$ is bounded within a positive range; see Section 3 . The initial value of $\psi_{i}$ defined in (12) is increased successively by a chosen constant factor $\beta>1$, and $\boldsymbol{p}^{(i+1)}$ is recalculated from (11), until the following sufficient decrease criterion is satisfied:

$$
Q\left(\boldsymbol{p}^{(i+1)}\right) \leq Q\left(\boldsymbol{p}^{(i)}\right)-\frac{\psi_{i} \sigma_{0}}{2}\left\|\boldsymbol{p}^{(i+1)}-\boldsymbol{p}^{(i)}\right\|^{2},
$$

for some specified $\sigma_{0} \in(0,1)$. Note that the evaluation of $Q(\boldsymbol{p})$ needed in (13) can be done efficiently through a parallel computation of $(\nabla \hat{f}(\boldsymbol{p})+\nabla \tilde{f}(\boldsymbol{w}))^{T} \boldsymbol{p} / 2$ plus the $\hat{g}(\boldsymbol{p})$ term. From the boundedness of $H$, one can easily prove that (13) is satisfied after a finite number of increases of $\psi_{i}$, as we will show in Section 3. In our algorithm, SpaRSA runs until either a fixed number of iterations is reached, or when some certain inner stopping condition for optimizing (2) is satisfied.
For general $H$, the computational bottleneck of $\nabla \hat{f}$ would take $O\left(d^{2}\right)$ operations to compute the $H \boldsymbol{p}^{(i)}$ term. However, for our LBFGS choice of $H_{k}$, this cost is reduced to $O\left(m d+m^{2}\right)$ by utilizing the matrix structure, as shown in the following formula:

$\nabla \hat{f}(\boldsymbol{p})=\nabla \tilde{f}(\boldsymbol{w})+H \boldsymbol{p}=\nabla \tilde{f}(\boldsymbol{w})+\gamma \boldsymbol{p}-U_{t}\left(M_{t}^{-1}\left(U_{t}^{T} \boldsymbol{p}\right)\right)$.

The computation of (14) can be parallelized, by first parallelizing computation of the inner product $U_{t}^{T} \boldsymbol{p}^{(i)}$ via the formula

$$
\sum_{k=1}^{K}\left(U_{t}\right)_{J_{k},:}^{T} \boldsymbol{p}_{J_{k}}^{(i)}
$$

with $O(m)$ communication. (We implement the parallel inner products as described in Section 2.3.) We either construct the whole vector $\mathbf{u}$ in (10) on all machines, or let each machine compute a subvector of $\mathbf{u}$ in (10). The former scheme is most suitable when $g$ is non-separable, but the latter has a lower computational burden per machine, in cases for which it is feasible to apply. We describe the latter scheme in more detail. The $k$ th machine locally computes $\boldsymbol{p}_{J_{k}}^{(i)}$ without communicating the whole vector. Then at each iteration of SpaRSA, partial inner products between $\left(U_{t}\right)_{J_{k}, \text { : }}$ and $\boldsymbol{p}_{J_{k}}^{(i)}$ can be computed locally, and the results are assembled with one $O(m)$ communication. This technique also suggests a spatial advantage of our method: The rows of $S_{t}$ and $Y_{t}$ can be stored in a distributed manner consistent with the subvector partition. This approach incurs $O(m)$ communication cost per SpaRSA iteration, with the computational cost reduced from $O(m d)$ to $O(m d / K)$ per machine. Since both the $O(m)$ communication cost and the $O(m d / K)$ computational cost are inexpensive when $m$ is small, in comparison to the computation of $\nabla \tilde{f}$, one can afford to conduct multiple iterations of SpaRSA at every main iteration. Note that the latency incurred at every communication as discussed in (3) can be capped by setting a maximum iteration limit for SpaRSA. Finally, after the SpaRSA procedure terminates, all machines conduct one $O(d)$ communication to gather the update step $\boldsymbol{p}$.

The distributed implementation of SpaRSA for solving (2) is summarized in Algorithm 1.

\subsection{Line Search}

After obtaining an update direction $\boldsymbol{p}^{k}$ by approximately minimizing $Q_{H_{k}}\left(\cdot ; \boldsymbol{w}_{k}\right)$, a line search procedure is usually needed to find a step size $\alpha_{k}$ that ensures sufficient decrease in the objective value. We follow Tseng and Yun [21] by using a modified-Armijo-type backtracking line search to find a suitable step size $\alpha$. Given the current iterate $\boldsymbol{w}$, the update direction $\boldsymbol{p}$, and parameters $\sigma_{1}, \theta \in(0,1)$, we set

$$
\Delta:=\nabla \tilde{f}(\boldsymbol{w})^{T} \boldsymbol{p}+g(\boldsymbol{w}+\boldsymbol{p})-g(\boldsymbol{w})
$$

and pick the step size as the largest of $\theta^{0}, \theta^{1}, \ldots$ satisfying

$$
F(\boldsymbol{w}+\alpha \boldsymbol{p}) \leq F(\boldsymbol{w})+\alpha \sigma_{1} \Delta .
$$

The computation of $\Delta$ can again be done in a distributed manner. First, $X_{k}^{T} \boldsymbol{p}$ can be computed locally on each machine, 


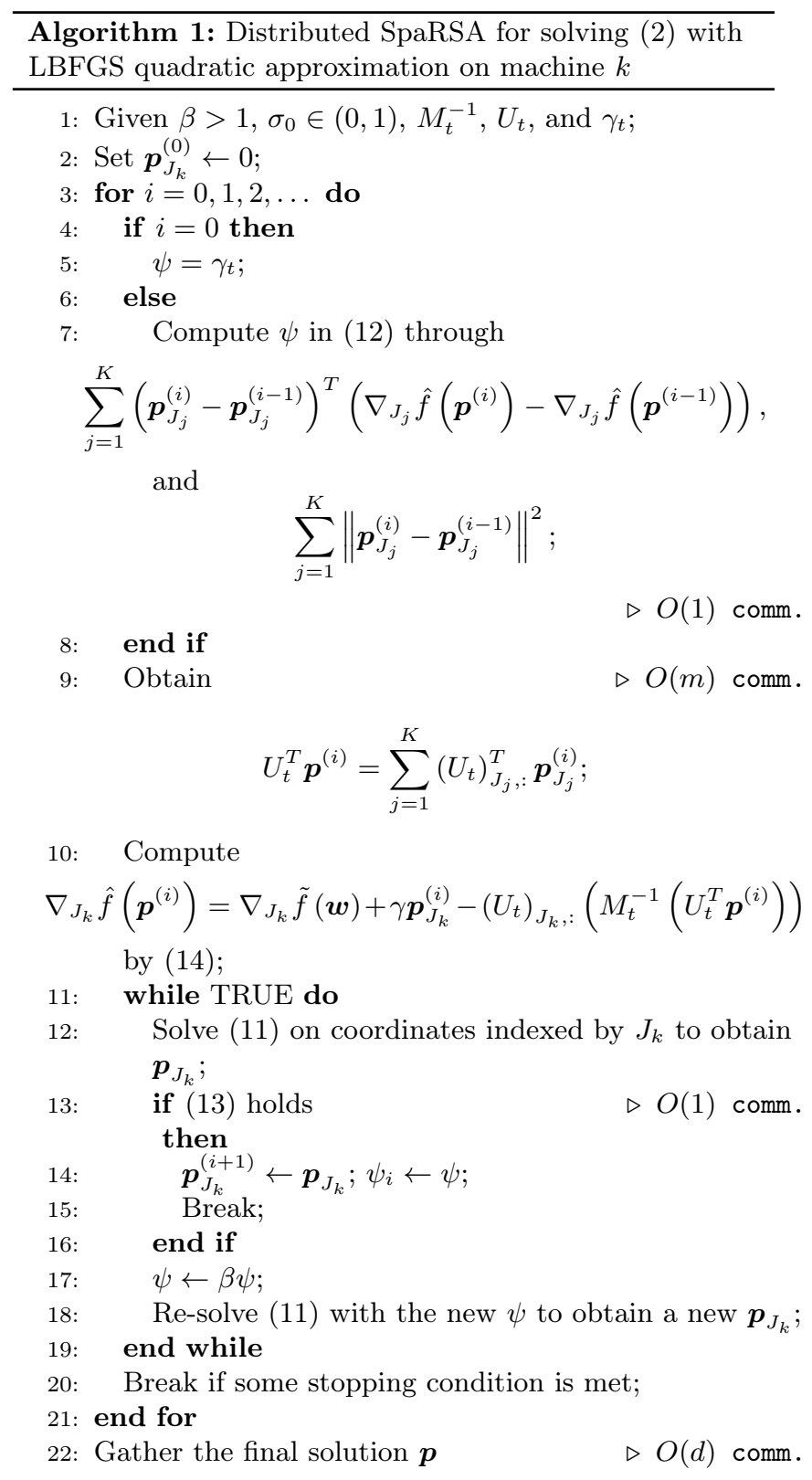

then the first term in (15) is obtained by sending a scalar over the network. When $g$ is block-separable, its computation can also be distributed across machines. The vector $X_{k}^{T} \boldsymbol{p}$ is then used to compute the left-hand side of (16) for arbitrary values of $\alpha$. Writing $X_{k}^{T}(\boldsymbol{w}+\alpha \boldsymbol{p})=\left(X_{k}^{T} \boldsymbol{w}\right)+\alpha\left(X_{k}^{T} \boldsymbol{p}\right)$, we see that once $X_{k}^{T} \boldsymbol{w}$ and $X_{k}^{T} \boldsymbol{p}$ are known, we can evaluate $X_{k}^{T}(\boldsymbol{w}+\alpha \boldsymbol{p})$ via an "axpy" operation (weighted sum of two vectors). Because $H_{t}$ defined in (5) attempts to approximate the real Hessian, the unit step $\alpha=1$ frequently satisfies (16), so we use the value 1 as the initial guess. Aside from the communication needed to compute the summation of the $f_{k}$ terms in the evaluation of $F$, the only other communication

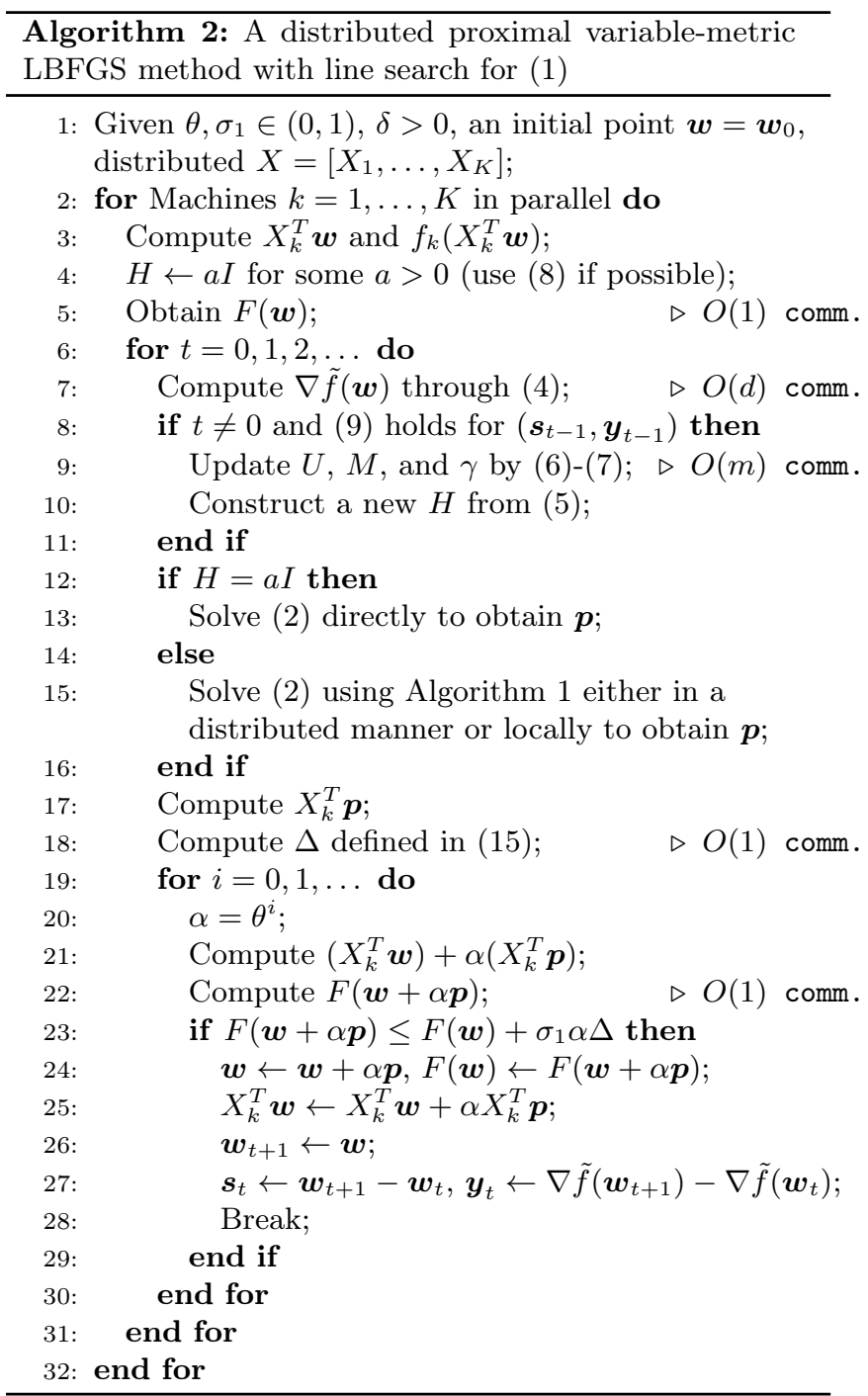

needed is to share the update direction $\boldsymbol{p}$ if (2) was solved in a distributed manner. Thus, two rounds of $O(d)$ communication are incurred per main iteration. Otherwise, if each machine solves the same subproblem (2) locally, then only one round of $O(d)$ communication is required.

Our distributed algorithm for (1) is summarized in Algorithm 2 .

\subsection{Cost Analysis}

We now summarize the costs of our algorithm. For the distributed version of Algorithm 1, each iteration costs

$$
O\left(\frac{d}{K}+\frac{m d}{K}+m^{2}\right)=O\left(\frac{m d}{K}+m^{2}\right)
$$

in computation and

$$
O(m+1 \times \text { number of times }(13) \text { is evaluated })
$$


in communication. In the next section, we will show that (13) is accepted in a constant number of times and thus the overall communication cost is $O(m)$.

For Algorithm 2, the computational cost per iteration is

$O\left(\frac{\# \mathrm{nnz}}{K}+\frac{n}{K}+d+\frac{m d}{K}+\frac{d}{K}\right)=O\left(\frac{\# \mathrm{nnz}}{K}+d+\frac{m d}{K}\right)$

where $\#$ nnz is the number of nonzero elements in $X$, and the communication cost is

$$
O(1+m+d)=O(d)
$$

We note that the costs of Algorithm 1 are dominated by those of Algorithm 2 if a fixed number of SpaRSA iterations is conducted every main iteration.

\section{COMMUNICATION COMPLEXITY}

The use of an iterative solver for the subproblem (2) generally results in an inexact solution. We first show that running SpaRSA for any fixed number of iterations guarantees a step $\boldsymbol{p}$ whose accuracy is sufficient to prove overall convergence.

Lemma 3.1. Using $H_{t}$ as defined in (5) with the safeguard mechanism (9) in (2), we have the following.

(1) There exist constants $c_{1} \geq c_{2}>0$ such that $c_{1} I \succeq H_{t} \succeq$ $c_{2} I$ for all main iterations. Moreover, $\left\|X^{T} X\right\| L \geq \gamma_{t} \geq$ $\delta$ for all $t>0$.

(2) The initial estimate of $\psi_{i}$ at every SpaRSA iteration is bounded within the range of $\left[\min \left\{c_{2}, \delta\right\}, \max \left\{c_{1},\left\|X^{T} X\right\| L\right\}\right]$, and the final accepted value $\psi_{i}$ is upper-bounded.

(3) SpaRSA is globally Q-linear convergent in solving (2). Therefore, there exists $\eta \in[0,1)$ such that if we run at least $S$ iterations of SpaRSA for all main iterations for any $S>0$, the approximate solution $\boldsymbol{p}$ satisfies

$$
-\eta^{S} Q^{*}=\eta^{S}\left(Q(0)-Q^{*}\right) \geq Q(\boldsymbol{p})-Q^{*},
$$

where $Q^{*}$ is the optimal objective of (2).

Lemma 3.1 establishes how the number of iterations of SpaRSA affects the inexactness of the subproblem solution. Given this measure, we can leverage the results developed in Lee and Wright [9] to obtain iteration complexity guarantees for our algorithm. Since in our algorithm, communication complexity scales linearly with iteration complexity, this guarantee provides a bound on the amount of communication. In particular, our method communicates $O(d+m S)$ bytes per iteration (where $S$ is the number of SpaRSA iterations used, as in Lemma 3.1) and the second term can usually be ignored for small $m$.

We show next that the step size generated by our line search procedure in Algorithm 2 is lower bounded by a positive value.

LEMmA 3.2. If SpaRSA is run at least $S$ iterations in solving (2), the corresponding $\Delta$ defined in (15) satisfies

$$
\Delta \leq-\frac{c_{2}\|d\|^{2}}{\left(1+\eta^{S / 2}\right)}
$$

Moreover, the backtracking subroutine in Algorithm 2 terminates in finite steps and produces a step size

$$
\alpha \geq \bar{\alpha} \geq \min \left\{1, \frac{2 \theta\left(1-\sigma_{1}\right) c_{2}}{\left\|X^{T} X\right\| L\left(1+\eta^{S / 2}\right)}\right\} .
$$

This result is just a worst-case guarantee; in practice we often observe that the line search procedure terminates with $\alpha=1$ for our choice of $H$, as we see in our experiments.

Next, we analyze communication complexity of Algorithm 2.

Theorem 3.3. If we apply Algorithm 2 to solve (1), and Algorithm 1 is run for $S$ iterations at each main iteration, then the following claims hold.

- Suppose that the following variant of strong convexity holds: There exists $\mu>0$ such that for any $\boldsymbol{w}$ and any $a \in[0,1]$, we have

$$
\begin{aligned}
& F\left(a \boldsymbol{w}+(1-a) P_{\Omega}(\boldsymbol{w})\right) \\
\leq & a F(\boldsymbol{w})+(1-a) F^{*}-\frac{\mu a(1-a)}{2}\left\|\boldsymbol{w}-P_{\Omega}(\boldsymbol{w})\right\|^{2},
\end{aligned}
$$

where $F^{*}$ is the optimal objective value of (1), $\Omega$ is the solution set, and $P_{\Omega}$ is the projection onto this set. Then Algorithm 2 converges globally at a Q-linear rate. That is,

$$
\frac{F\left(\boldsymbol{w}_{t+1}\right)-F^{*}}{F\left(\boldsymbol{w}_{t}\right)-F^{*}} \leq 1-\frac{\bar{\alpha} \sigma_{1}\left(1-\eta^{S}\right) \mu}{\mu+c_{1}}, \quad \forall t .
$$

Therefore, to get an approximate solution of (1) that is $\epsilon$ accurate in the sense of objective value, we need to perform at most

$$
O\left(\frac{\mu+c_{1}}{\mu \sigma_{1} \bar{\alpha}\left(1-\eta^{S}\right)} \log \frac{1}{\epsilon}\right)
$$

rounds of $O(d)$ communication.

- When $F$ is convex, and the level set defined by $\boldsymbol{w}_{0}$ is bounded, define

$$
R_{0}:=\sup _{\boldsymbol{w}: F(\boldsymbol{w}) \leq F\left(\boldsymbol{w}_{0}\right)}\left\|\boldsymbol{w}-P_{\Omega}(\boldsymbol{w})\right\| .
$$

Then we obtain the following expressions for rate of convergence of the objective value.

(1) When $F\left(\boldsymbol{w}_{t}\right)-F^{*} \geq c_{1} R_{0}^{2}$,

$$
\frac{F\left(\boldsymbol{w}_{t+1}\right)-F^{*}}{F\left(\boldsymbol{w}_{t}\right)-F^{*}} \leq 1-\frac{\left(1-\eta^{S}\right) \sigma_{1} \bar{\alpha}}{2} .
$$

(2) Otherwise, we have globally for all t that

$$
\frac{F\left(\boldsymbol{w}_{t}\right)-F^{*}}{2} \leq \frac{c_{1} R_{0}^{2}+F\left(\boldsymbol{w}_{0}\right)-F^{*}}{\sigma_{1} t\left(1-\eta^{S}\right) \bar{\alpha}} .
$$

This implies a communication complexity of

$$
\begin{cases}O\left(\frac{2}{\left(1-\eta^{S}\right) \sigma_{1} \bar{\alpha}} \log \frac{1}{\epsilon}\right) & \text { if } \epsilon \geq c_{1} R_{0}^{2}, \\ \frac{2\left(c_{1} R_{0}^{2}+F\left(\boldsymbol{w}_{0}\right)-F^{*}\right)}{\sigma_{1}\left(1-\eta^{S}\right) \bar{\alpha} \epsilon} & \text { else. }\end{cases}
$$

- If $F$ is non-convex, the norm of the proximal gradient steps

$$
G_{t}:=\arg \min _{\boldsymbol{p}} \nabla f\left(\boldsymbol{w}_{t}\right)^{T} \boldsymbol{p}+\frac{\|\boldsymbol{p}\|^{2}}{2}+g\left(\boldsymbol{w}_{t}+\boldsymbol{p}\right)
$$


converge to zero at a rate of $O(1 / \sqrt{t})$ in the following sense:

$\min _{0 \leq i \leq t}\left\|G_{i}\right\|^{2} \leq \frac{F\left(\boldsymbol{w}_{0}\right)-F^{*}}{\gamma(t+1)} \frac{c_{1}^{2}\left(1+\frac{1}{c_{2}}+\sqrt{1-\frac{2}{c_{1}}+\frac{1}{c_{2}^{2}}}\right)^{2}}{2 c_{2} \bar{\alpha}\left(1-\eta^{S}\right)}$.

Note that it is known that the norm of $G_{t}$ is zero if and only if $\boldsymbol{w}_{t}$ is a stationary point [9], so this measure serves as a first-order optimality condition.

Our computational experiments cover the case of $F$ convex; exploration of the method on nonconvex $F$ is left for future work.

\section{RELATED WORKS}

The framework of using (2) to generate update directions for optimizing (1) has been discussed in existing works with different choices of $H$, but always in the single-core setting. Lee et al. [10] focused on using $\nabla^{2} \tilde{f}$ as $H$, and proved local convergence results under certain additional assumptions. In their experiment, they used AG to solve (2). However, in distributed environments, using $\nabla^{2} \tilde{f}$ as $H$ incurs an $O(d)$ communication cost per AG iteration in solving (2), because computation of the term $\nabla^{2} \tilde{f}(\boldsymbol{w}) \boldsymbol{p}=X \nabla^{2} f\left(X^{T} \boldsymbol{w}\right) X^{T} \boldsymbol{p}$ requires one allreduce operation to calculate a weighted sum of the columns of $X$.

Scheinberg and Tang [18] and Ghanbari and Scheinberg [6] showed global convergence rate results for a method based on (2) with bounded $H$, and suggested using randomized coordinate descent to solve (2). In the experiments of these two works, they used the same choice of $H$ as we do in this paper, with CD as the solver for (2), which is well suited to their single-machine setting. Aside from our extension to the distributed setting and the use of SpaRSA, the third major difference between their algorithm and ours is that they do not conduct line search on the step size. Instead, when the obtained solution with a unit step size does not result in sufficient objective value decrease, they add a scaled identity matrix to $H$ and solve (2) again starting from $\boldsymbol{p}^{(0)}=0$. The cost of repeatedly solving (2) from scratch can be high, which results in an algorithm with higher overall complexity. This potential inefficiency is exacerbated further by the inefficiency of coordinate descent in the distributed setting.

Our method can be considered as a special case of the algorithmic framework in Bonettini et al. [3], Lee and Wright [9], which both focus on analyzing the theoretical guarantees under various conditions. In the experiments of Bonettini et al. [3], $H$ is obtained from the diagonal entries of $\nabla^{2} \tilde{f}$, making the subproblem (2) easy to solve, but this simplification does not take full advantage of curvature information. Although our theoretical convergence analysis follows directly from Lee and Wright [9], that paper does not provide details of experimental results or implementation, and its analysis focuses on general $H$ rather than the LBFGS choice we use here.

Some methods consider solving (1) in a distributed environment where $X$ is partitioned feature-wise (i.e. along rows instead of columns). There are two potential disadvantages of
Table 1: Data statistics.

\begin{tabular}{l|rr|r} 
Data set & $n$ & $d$ & \#nonzeros \\
\hline news & 19,996 & $1,355,191$ & $9,097,916$ \\
epsilon & 400,000 & 2,000 & $800,000,000$ \\
webspam & 350,000 & $16,609,143$ & $1,304,697,446$ \\
avazu-site & $25,832,830$ & 999,962 & $387,492,144$
\end{tabular}

this approach. First, new data points can easily be assigned to one of the machines in our approach, whereas in the featurewise approach, the features of all new points would need to be distributed around the machines. Second, local curvature information is obtained, so the update direction can be poor if the data is distributed nonuniformly across features. (Data is more likely to be distributed evenly across instances than across features.) In the extreme case in which each machine contains only one row of $X$, only the diagonal entries of the Hessian can be obtained locally, so the method reduces to a scaled version of proximal gradient.

\section{NUMERICAL EXPERIMENTS}

We investigate the empirical performance of Algorithm 2 in solving $\ell_{1}$-regularized logistic regression problems. The code used in our experiment is available at http://github. com/leepei/dplbfgs/. Given training data points $\left(\boldsymbol{x}_{i}, y_{i}\right) \in$ $\mathbb{R}^{d} \times\{-1,1\}$ for $i=1, \ldots, n$, the objective function is

$$
F(\boldsymbol{w})=C \sum_{i=1}^{n} \log \left(1+e^{-y_{i} \boldsymbol{x}_{i}^{T} \boldsymbol{w}}\right)+\|\boldsymbol{w}\|_{1}
$$

where $C>0$ is a parameter prespecified to trade-off between the loss term and the regularization term. We fix $C$ to 1 for simplicity in our experiments. We consider the publicly available binary classification data sets listed in Table $1^{1}$, and partitioned the instances evenly across machines.

The parameters of our algorithm were set as follows: $\theta=$ $0.5, \beta=2, \sigma_{0}=10^{-2}, \sigma_{1}=10^{-4}, m=10, \delta=10^{-10}$. The parameters in SpaRSA follow the setting in [23], $\theta$ is set to halve the step size each time, the value of $\sigma_{0}$ follows the default experimental setting of [7], $\delta$ is set to a small enough number, and $m=10$ is a common choice for LBFGS.

We ran our experiments on a local cluster of 16 machines running MPICH2, and all algorithms are implemented in $\mathrm{C} / \mathrm{C}++$. The inversion of $M$ defined in (6) is performed through LAPACK [1]. The comparison criteria are the relative objective error $\left(F(\boldsymbol{w})-F^{*}\right) / F^{*}$, versus either the amount communicated (divided by $d$ ) or the overall running time. The former criterion is useful in estimating the performance in environments in which communication cost is extremely high.

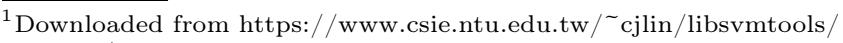
datasets/.
} 
Table 2: Different stopping conditions of SpaRSA as an approximate solver for (2). We show required amount of communication (divided by $d$ ) and running time (in seconds) to reach $F\left(\boldsymbol{w}_{t}\right)-F^{*} \leq 10^{-3} F^{*}$.

\begin{tabular}{l|r|r|r} 
Data set & $\epsilon_{1}$ & Communication & Time \\
\hline \multirow{3}{*}{ news20 } & $10^{-1}$ & 28 & 11 \\
& $10^{-2}$ & 25 & 11 \\
& $10^{-3}$ & 23 & 14 \\
\hline \multirow{3}{*}{ epsilon } & $10^{-1}$ & 144 & 45 \\
& $10^{-2}$ & 357 & 61 \\
& $10^{-3}$ & 687 & 60 \\
\hline \multirow{3}{*}{ webspam } & $10^{-1}$ & 452 & 3254 \\
& $10^{-2}$ & 273 & 1814 \\
& $10^{-3}$ & 249 & 1419
\end{tabular}

\subsection{Effect of Inexactness in the Subproblem Solution}

We first examine how the degree of inexactness of the approximate solution of subproblems (2) affects the convergence of the overall algorithm. Instead of treating SpaRSA as a steadily linearly converging algorithm, we take it as an algorithm that sometimes decreases the objective much faster than the worst-case guarantee, thus an adaptive stopping condition is used. In particular, we terminate Algorithm 1 when the norm of the current update step is smaller than $\epsilon_{1}$ times that of the first update step, for some prespecified $\epsilon_{1}>0$. From the proof of Lemma 3.1, the norm of the update step bounds the value of $Q(\boldsymbol{p})-Q^{*}$ both from above and from below, and thus serves as a good measure of the solution precision. In Table 2, we compare runs with the values $\epsilon_{1}=10^{-1}, 10^{-2}, 10^{-3}$. For the datasets news 20 and webspam, it is as expected that tighter solution of (2) results in better updates and hence lower communication cost. This may not result in a longer convergence time. As for the dataset epsilon, which has a smaller data dimension $d$, the $O(m)$ communication cost per SpaRSA iteration for calculating $\nabla \tilde{f}$ is significant in comparison. In this case, setting a tighter stopping criteria for SpaRSA can result in higher communication cost and longer running time.

In Table 3, we show the distribution of the step sizes over the main iterations, for the same set of values of $\epsilon_{1}$. As we discussed in Section 3, although the smallest $\alpha$ can be much smaller than one, the unit step is usually accepted. Therefore, although the worst-case communication complexity analysis is dominated by the smallest step encountered, the practical behavior is much better.

\subsection{Comparison with Other Methods}

Now we compare our method with two state-of-the-art distributed algorithms for (1). In addition to a proximal-gradienttype method that can be used to solve general (1) in distributed environments easily, we also include one solver specifically designed for $\ell_{1}$-regularized problems in our comparison. These methods are:
Table 3: Step size distributions.

\begin{tabular}{l|r|r|r} 
Data set & $\epsilon_{1}$ & percent of $\alpha=1$ & smallest $\alpha$ \\
\hline \multirow{3}{*}{ news20 } & $10^{-1}$ & $95.5 \%$ & $2^{-3}$ \\
& $10^{-2}$ & $95.5 \%$ & $2^{-4}$ \\
& $10^{-3}$ & $95.5 \%$ & $2^{-3}$ \\
\hline \multirow{3}{*}{ epsilon } & $10^{-1}$ & $96.8 \%$ & $2^{-5}$ \\
& $10^{-2}$ & $93.4 \%$ & $2^{-6}$ \\
& $10^{-3}$ & $91.2 \%$ & $2^{-3}$ \\
\hline \multirow{3}{*}{ webspam } & $10^{-1}$ & $98.5 \%$ & $2^{-3}$ \\
& $10^{-2}$ & $97.6 \%$ & $2^{-2}$ \\
& $10^{-3}$ & $97.2 \%$ & $2^{-2}$
\end{tabular}

- DPLBFGS: our Distributed Proximal LBFGS approach. We fix $\epsilon_{1}=10^{-2}$ in this experiment.

- SPARSA [23]: the method described in Section 2.4, but applied directly to (1).

- OWLQN [2]: an orthant-wise quasi-Newton method specifically designed for $\ell_{1}$-regularized problems. We fix $m=10$ in the LBFGS approximation.

We implement all methods in $\mathrm{C} / \mathrm{C}++$ and MPI. Note that the AG method [17] can also be used, but its empirical performance has been shown to be similar to SpaRSA [24] and it requires strong convexity and Lipschitz parameters to be estimated, which induces an additional cost. A further examination on different values of $m$ indicates that convergence speed of our method improves with larger $m$, while in OWLQN, larger $m$ usually does not lead to better results. We use the same value of $m$ for both methods and choose a value that favors OWLQN.

The results are provided in Figure 1. Our method is always the fastest in both criteria. For epsilon, our method is orders of magnitude faster, showing that correctly using the curvature information of the smooth part is indeed beneficial in reducing the communication complexity.

It is possible to include specific heuristics for $\ell_{1}$-regularized problems, such as those applied in Yuan et al. [25], Zhong et al. [27], to further accelerate our method, and the exploration on this direction is an interesting topic for future work.

\section{CONCLUSIONS}

In this work, we propose a practical and communicationefficient distributed algorithm for solving general regularized nonsmooth ERM problems. Our algorithm enjoys fast performance both theoretically and empirically and can be applied to a wide range of ERM problems. It is possible to extend our approach for solving the distributed dual ERM problem with a strongly convex primal regularizer, and we expect our framework to outperform state of the art, which only uses block-diagonal parts of the Hessian that can be computed locally. These topics are left for future work.

\section{ACKNOWLEDGEMENT}

This work was supported by NSF Awards IIS-1447449, 1628384, 1634597, and 1740707; AFOSR Award FA9550-13-1-0138; and 


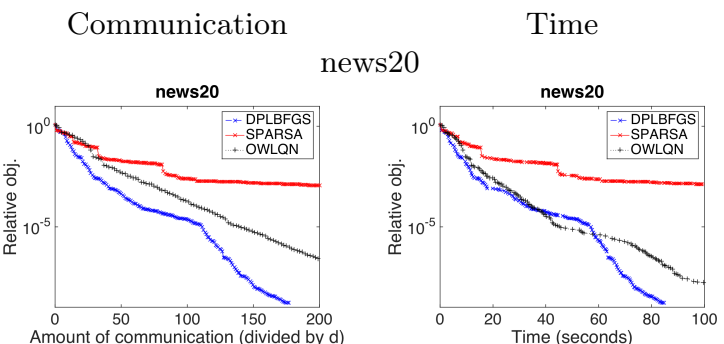

epsilon
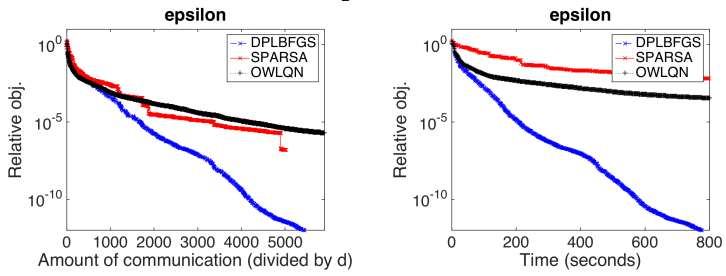

webspam
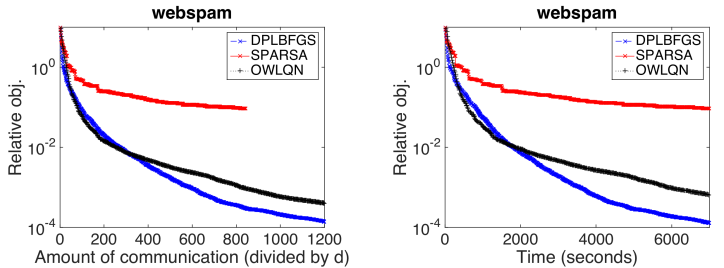

avazu-site
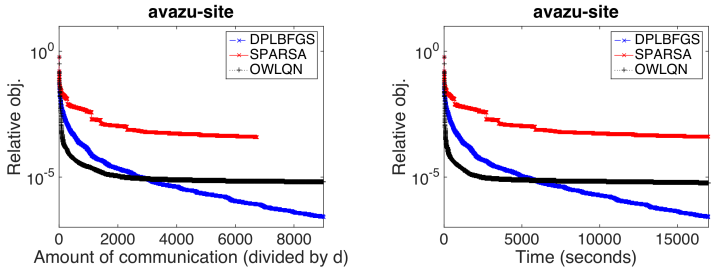

Figure 1: Comparison between different methods for $\ell_{1}$-regularized logistic regression in terms of relative objective difference to the optimum. Left: communication (divided by $d$ ); right: running time (in seconds).

Subcontract 3F-30222 from Argonne National Laboratory. The authors thank En-Hsu Yen for fruitful discussions.

\section{REFERENCES}

[1] Edward Anderson, Zhaojun Bai, Christian Bischof, L Susan Blackford, James Demmel, Jack Dongarra, Jeremy Du Croz, Anne Greenbaum, Sven Hammarling, Alan McKenney, et al. 1999. LAPACK Users' guide. SIAM.

[2] Galen Andrew and Jianfeng Gao. 2007. Scalable Training of L1Regularized Log-Linear Models. In International Conference on Machine Learning.

[3] Silvia Bonettini, Ignace Loris, Federica Porta, and Marco Prato. 2016. Variable metric inexact line-search-based methods for nonsmooth optimization. SIAM journal on optimization 26, 2 (2016), 891-921.

[4] Richard H. Byrd, Jorge Nocedal, and Robert B. Schnabel. 1994 Representations of quasi-Newton matrices and their use in limited memory methods. Mathematical Programming 63, 1-3 (1994), $129-156$.
[5] Ernie Chan, Marcel Heimlich, Avi Purkayastha, and Robert Van De Geijn. 2007. Collective communication: theory, practice, and experience. Concurrency and Computation: Practice and Experience 19, 13 (2007), 1749-1783.

[6] Hiva Ghanbari and Katya Scheinberg. 2016. Proximal QuasiNewton Methods for Convex Optimization. Technical Report. arXiv:1607.03081.

[7] Ching-pei Lee, Po-Wei Wang, Weizhu Chen, and Chih-Jen Lin. 2017. Limited-memory Common-directions Method for Distributed Optimization and its Application on Empirical Risk Minimization. In Proceedings of SIAM International Conference on Data Mining.

[8] Ching-pei Lee and Stephen J. Wright. 2017. Using Neural Networks to Detect Line Outages from PMU Data. Technical Report. arXiv:1710.05916.

[9] Ching-pei Lee and Stephen J. Wright. 2018. Inexact Successive Quadratic Approximation for Regularized Optimization. Technical Report.

[10] Jason D. Lee, Yuekai Sun, and Michael A. Saunders. 2014. Proximal Newton-type methods for minimizing composite functions. SIAM Journal on Optimization 24, 3 (2014), 1420-1443.

11] Dong-Hui Li and Masao Fukushima. 2001. On the global convergence of the BFGS method for nonconvex unconstrained optimization problems. SIAM Journal on Optimization 11, 4 (2001), 1054-1064.

[12] Chieh-Yen Lin, Cheng-Hao Tsai, Ching-Pei Lee, and Chih-Jen Lin. 2014. Large-scale logistic regression and linear support vector machines using Spark. In Proceedings of the IEEE International Conference on Big Data. 519-528.

[13] Dong C. Liu and Jorge Nocedal. 1989. On the limited memory BFGS method for large scale optimization. Mathematical programming 45, 1 (1989), 503-528.

[14] Xiangrui Meng, Joseph Bradley, Burak Yavuz, Evan Sparks, Shivaram Venkataraman, Davies Liu, Jeremy Freeman, DB Tsai, Manish Amde, Sean Owen, et al. 2016. MLlib: Machine learning in Apache Spark. Journal of Machine Learning Research 17, 1 (2016), 1235-1241.

[15] Message Passing Interface Forum. 1994. MPI: a message-passing interface standard. International Journal on Supercomputer Applications 8, 3/4 (1994).

[16] Yu Nesterov. 1983. A method of solving a convex programming problem with convergence rate $O\left(1 / k^{2}\right)$. Soviet Mathematics Doklady 27 (1983), 372-376.

[17] Yu Nesterov. 2013. Gradient methods for minimizing composite functions. Mathematical Programming 140, 1 (2013), 125-161.

[18] Katya Scheinberg and Xiaocheng Tang. 2016. Practical inexact proximal quasi-Newton method with global complexity analysis. Mathematical Programming 160, 1-2 (2016), 495-529.

[19] Mark Schmidt, Nicolas Roux, and Francis Bach. 2011. Convergence rates of inexact proximal-gradient methods for convex optimization. In Advances in neural information processing systems. 1458-1466.

[20] Ohad Shamir, Nati Srebro, and Tong Zhang. 2014. Communication-efficient distributed optimization using an approximate Newton-type method. In International conference on machine learning.

[21] Paul Tseng and Sangwoon Yun. 2009. A coordinate gradient descent method for nonsmooth separable minimization. Mathematical Programming 117, 1 (2009), 387-423.

[22] Po-Wei Wang, Ching-pei Lee, and Chih-Jen Lin. 2016. The Common Directions Method for Regularized Empirical Loss Minimization. Technical Report. National Taiwan University.

[23] Stephen J. Wright, Robert D. Nowak, and Mário A. T. Figueiredo. 2009. Sparse reconstruction by separable approximation. IEEE Transactions on Signal Processing 57, 7 (2009), 2479-2493.

[24] Junfeng Yang and Yin Zhang. 2011. Alternating direction algorithms for $\ell 1$-problems in compressive sensing. SIAM journal on scientific computing 33, 1 (2011), 250-278.

[25] Guo-Xun Yuan, Chia-Hua Ho, and Chih-Jen Lin. 2012. An Improved GLMNET for L1-regularized Logistic Regression. Journal of Machine Learning Research 13 (2012), 1999-2030.

[26] Yuchen Zhang and Xiao Lin. 2015. DiSCO: Distributed Optimization for Self-Concordant Empirical Loss. In International Conference on Machine Learning.

[27] Kai Zhong, Ian En-Hsu Yen, Inderjit S. Dhillon, and Pradeep K. Ravikumar. 2014. Proximal quasi-Newton for computationally intensive 11 -regularized $M$-estimators. In Advances in Neural 
Information Processing Systems.

[28] Yong Zhuang, Wei-Sheng Chin, Yu-Chin Juan, and Chih-Jen Lin. 2015. Distributed Newton method for regularized logistic regression. In Proceedings of the Pacific-Asia Conference on Knowledge Discovery and Data Mining (PAKDD).

\section{A PROOFS}

We provide proof of Lemma 3.1 in this section. The rest of Section 3 directly follows the results in Lee and Wright [9] by noting that $\nabla \tilde{f}(\boldsymbol{w})$ is $\left\|X^{T} X\right\| L$-Lipschitz continuous, and are therefore omitted.

Proof of Lemma 3.1. We prove the three results separately.

(1) The boundedness of $H_{t}$ directly follow from the results in Li and Fukushima [11]. A more detailed proof can be found in, for example, Lee and Wright [8, Appendix E]. The lower bound of $\gamma_{t}$ is directly through (9), and the upper bound is from the Lipschitz continuity of $\nabla \tilde{f}$.

(2) By directly expanding $\nabla \hat{f}$, we have that for any $\boldsymbol{p}_{1}, \boldsymbol{p}_{2}$,

$$
\begin{aligned}
\nabla \hat{f}\left(\boldsymbol{p}_{1}\right)-\nabla \hat{f}\left(\boldsymbol{p}_{2}\right) & =\nabla \tilde{f}(\boldsymbol{w})+H \boldsymbol{p}_{1}-\left(\nabla \tilde{f}(\boldsymbol{w})+H \boldsymbol{p}_{2}\right) \\
& =H\left(\boldsymbol{p}_{1}-\boldsymbol{p}_{2}\right) .
\end{aligned}
$$

Therefore, we have

$$
\frac{\left(\nabla \hat{f}\left(\boldsymbol{p}_{1}\right)-\nabla \hat{f}\left(\boldsymbol{p}_{2}\right)\right)^{T}\left(\boldsymbol{p}_{1}-\boldsymbol{p}_{2}\right)}{\left\|\boldsymbol{p}_{1}-\boldsymbol{p}_{2}\right\|^{2}}=\frac{\left\|\boldsymbol{p}_{1}-\boldsymbol{p}_{2}\right\|_{H}^{2}}{\left\|\boldsymbol{p}_{1}-\boldsymbol{p}_{2}\right\|^{2}} \in\left[c_{2}, c_{1}\right]
$$

for bounding $\psi_{i}$ for $i>0$, and the bound for $\psi_{0}$ is directly from the bounds of $\gamma_{t}$. The combined bound is therefore $\left[\min \left\{c_{2}, \delta\right\}, \max \left\{c_{1},\left\|X^{T} X\right\| L\right\}\right]$. Next, we show that the final $\psi_{i}$ is always upper-bounded. The right-hand side of (11) is equivalent to the following:

$\arg \min _{\boldsymbol{d}} \hat{Q}_{\psi_{i}}(\boldsymbol{d}):=\nabla \hat{f}\left(\boldsymbol{p}^{(i)}\right)^{T} \boldsymbol{d}+\frac{\psi_{i}\|\boldsymbol{d}\|^{2}}{2}+\hat{g}(\boldsymbol{d}+\boldsymbol{p})-\hat{g}(\boldsymbol{p})$.

Denote the optimal solution by $\boldsymbol{d}^{*}$, then we have $\boldsymbol{p}^{(i+1)}=\boldsymbol{p}^{(i)}+\boldsymbol{d}^{*}$. Because $H$ is upper-bounded by $c_{1}$, we have that $\nabla \hat{f}$ is $c_{1}$-Lipschitz continuous. Therefore, using Lemma 12 of Lee and Wright [9], we get

$$
\hat{Q}_{\psi_{i}}\left(\boldsymbol{d}^{*}\right) \leq-\frac{\psi_{i}}{2}\left\|\boldsymbol{d}^{*}\right\|^{2}
$$

We then have from $c_{1}$-Lipschitz continuity of $\nabla \hat{f}$ that

$$
\begin{gathered}
Q\left(\boldsymbol{p}^{(i+1)}\right)-Q\left(\boldsymbol{p}^{(i)}\right) \\
\leq \nabla \hat{f}\left(\boldsymbol{p}^{(i)}\right)^{T}\left(\boldsymbol{p}^{(i+1)}-\boldsymbol{p}^{(i)}\right)+\frac{c_{1}}{2}\left\|\boldsymbol{p}^{(i+1)}-\boldsymbol{p}^{(i)}\right\|^{2} \\
\quad+\hat{g}\left(\boldsymbol{p}^{(i+1)}\right)-\hat{g}\left(\boldsymbol{p}^{(i)}\right) \\
\stackrel{(25)}{=} \hat{Q}_{\psi_{i}}\left(\boldsymbol{d}^{*}\right)-\frac{\psi_{i}}{2}\left\|\boldsymbol{d}^{*}\right\|^{2}+\frac{c_{1}}{2}\left\|\boldsymbol{d}^{*}\right\|^{2} \\
\stackrel{(26)}{\leq}\left(\frac{c_{1}}{2}-\psi_{i}\right)\left\|\boldsymbol{d}^{*}\right\|^{2} .
\end{gathered}
$$

Therefore, whenever

$$
\frac{c_{1}}{2}-\psi_{i} \leq-\frac{\sigma_{0} \psi_{i}}{2},
$$

(13) holds. This is equivalent to

$$
\psi_{i} \geq \frac{c_{1}}{2-\sigma_{0}} .
$$

Since $\sigma_{0} \in(0,1)$, we must have $c_{1} /\left(2-\sigma_{0}\right) \in\left(c_{1} / 2, c_{1}\right)$, Note that the initialization of $\psi_{i}$ is upper-bounded by $c_{1}$ for all $i>1$, so the final $\psi_{i}$ is upper bounded by $2 c_{1}$. Together with the first iteration that we start with $\psi_{0}=\gamma_{t}$, we have that $\psi_{i}$ are always upper-bounded by $\max \left\{2 c_{1}, \gamma_{t}\right\}$, and we have already proven $\gamma_{t}$ is upper-bounded by $\left\|X^{T} X\right\| L$.

(3) We note that since $Q$ is $c_{2}$-strongly convex, the following condition holds.

$$
\frac{\min _{\boldsymbol{s} \in \nabla \hat{f}\left(\boldsymbol{p}^{(i+1)}\right)+\partial \hat{g}\left(\boldsymbol{p}^{(i+1)}\right)}\|\boldsymbol{s}\|^{2}}{2 c_{2}} \geq Q\left(\boldsymbol{p}^{(i+1)}\right)-Q^{*}
$$

On the other hand, from the optimality condition of (25), we have that

$$
\psi_{i} \boldsymbol{d}^{*}=\nabla \hat{f}\left(\boldsymbol{p}^{(i)}\right)+\boldsymbol{s}_{i+1},
$$

for some

$$
\boldsymbol{s}_{i+1} \in \partial \hat{g}\left(\boldsymbol{p}^{(i+1)}\right) \text {. }
$$

Therefore,

$$
Q\left(\boldsymbol{p}^{(i+1)}\right)-Q^{*}
$$

$$
\begin{aligned}
& \stackrel{(27)}{\leq} \frac{1}{2 c_{2}}\left\|\nabla \hat{f}\left(\boldsymbol{p}^{(i+1)}\right)-\nabla \hat{f}\left(\boldsymbol{p}^{(i)}\right)+\nabla \hat{f}\left(\boldsymbol{p}^{(i)}\right)+\boldsymbol{s}_{i+1}\right\|^{2} \\
& \stackrel{(28)}{\leq} \frac{1}{c_{2}}\left\|\nabla \hat{f}\left(\boldsymbol{p}^{(i+1)}\right)-\nabla \hat{f}\left(\boldsymbol{p}^{(i)}\right)\right\|^{2}+\left\|\psi_{i} \boldsymbol{d}^{*}\right\|^{2} \\
& \leq \frac{1}{c_{2}}\left(c_{1}^{2}+\psi^{2}\right)\left\|\boldsymbol{d}^{*}\right\|^{2}
\end{aligned}
$$

By combining (13) and (29), we obtain

$$
\begin{aligned}
Q\left(\boldsymbol{p}^{(i+1)}\right)-Q\left(\boldsymbol{p}^{(i)}\right) & \leq-\frac{\sigma_{0} \psi_{i}}{2}\left\|\boldsymbol{d}^{*}\right\|^{2} \\
& \leq-\frac{\sigma_{0} \psi_{i}}{2} \frac{c_{2}}{c_{1}^{2}+\psi^{2}}\left(Q\left(\boldsymbol{p}^{(i+1)}\right)-Q^{*}\right) .
\end{aligned}
$$

Rearranging the terms, we obtain

$\left(1+\frac{c_{2} \sigma_{0} \psi_{i}}{2\left(c_{1}^{2}+\psi^{2}\right)}\right)\left(Q\left(\boldsymbol{p}^{(i+1)}\right)-Q^{*}\right) \leq Q\left(\boldsymbol{p}^{(i)}\right)-Q^{*}$,

showing Q-linear convergence of SpaRSA, with

$$
\eta=\left(1+\frac{c_{2} \sigma_{0} \psi_{i}}{2\left(c_{1}^{2}+\psi_{i}^{2}\right)}\right)^{-1} \in[0,1]
$$

